\title{
Methylmalonic acid, vitamin B12, renal function, and risk of all-cause mortality in the general population: results from the prospective Lifelines-MINUTHE study
}

Ineke J. Riphagen ${ }^{1}$, Isidor Minović ${ }^{*}$, Dion Groothof ${ }^{2}$, Adrian Post $^{2}$, Manfred L. Eggersdorfer $^{3}$, Jenny E. Kootstra-Ros ${ }^{1}$, Martin H. de Borst ${ }^{2}$, Gerjan Navis ${ }^{2}$, Frits A. J. Muskiet ${ }^{1}$, Ido P. Kema' ${ }^{1}$, M. Rebecca Heiner-Fokkema ${ }^{1}$ and Stephan J. L. Bakker ${ }^{2}$

\begin{abstract}
Background: Methylmalonic acid (MMA) is best known for its use as a functional marker of vitamin B12 deficiency. However, MMA concentrations not only depend on adequate vitamin B12 status, but also relate to renal function and endogenous production of propionic acid. Hence, we aimed to investigate to what extent variation in MMA levels is explained by vitamin B12 and eGFR and whether MMA levels are associated with mortality if vitamin B12 and eGFR are taken into account.

Methods: A total of 1533 individuals (aged 60-75 years, 50\% male) were included from the Lifelines Cohort and Biobank Study. Individuals were included between 2006 and 2013, and the total follow-up time was 8.5 years.

Results: Median [IQR] age of the study population was 65 [62-69] years, 50\% was male. At baseline, median MMA concentration was 170 [138-216] nmol/L, vitamin B12 290 [224-362] pmol/L, and eGFR 84 [74-91] mL/min/1.73 m2. $\log _{2}$ vitamin $B 12, \log _{2}$ eGFR, age, and sex were significantly associated with $\log _{2}$ MMA in multivariable linear regression analyses (model $R^{2}=0.22$ ). After a total follow-up time of 8.5 years, 72 individuals had died. $\log _{2} \mathrm{MMA}$ levels were significantly associated with mortality (hazard ratio [HR] 1.67 [95\% Cl 1.25-2.22], $P<0.001$ ). Moreover, we found a significant interaction between MMA and eGFR with respect to mortality $\left(P_{\text {interaction }}<0.001\right)$.

Conclusions: Only 22\% of variation in MMA levels was explained by vitamin B12, eGFR, age, and sex, indicating that a large part of variation in MMA levels is attributable to other factors (e.g., catabolism, dietary components, or gut microbial production). Higher MMA levels are associated with an increased risk for mortality, independent of vitamin B12, eGFR, and sex. This association was more pronounced in individuals with impaired renal function.
\end{abstract}

\footnotetext{
* Correspondence: i.minovic@umcg.nl

${ }^{1}$ Department of Laboratory Medicine, University of Groningen, University

Medical Center Groningen, P.O. Box 30.001, 9700 RB Groningen, The

Netherlands

Full list of author information is available at the end of the article
}

C C The Author(s). 2020 Open Access This article is licensed under a Creative Commons Attribution 4.0 International License, which permits use, sharing, adaptation, distribution and reproduction in any medium or format, as long as you give appropriate credit to the original author(s) and the source, provide a link to the Creative Commons licence, and indicate if changes were made. The images or other third party material in this article are included in the article's Creative Commons licence, unless indicated otherwise in a credit line to the material. If material is not included in the article's Creative Commons licence and your intended use is not permitted by statutory regulation or exceeds the permitted use, you will need to obtain permission directly from the copyright holder. To view a copy of this licence, visit http://creativecommons.org/licenses/by/4.0/ The Creative Commons Public Domain Dedication waiver (http://creativecommons.org/publicdomain/zero/1.0/) applies to the data made available in this article, unless otherwise stated in a credit line to the data. 


\section{Background}

Methylmalonic acid (MMA) is a small water-soluble organic acid that is currently best known for its use as a functional marker for vitamin B12 deficiency [1]. Vitamin B12 is an essential cofactor for L-methylmalonylCoA mutase, which converts methylmalonyl-CoA into succinyl-CoA [2]. In vitamin B12 deficiency, the activity of L-methylmalonyl-CoA mutase is impaired, which results in the conversion of methylmalonyl-CoA into MMA [2].

However, concentrations of MMA are not only elevated in vitamin B12 deficiency, but are also known to increase during renal dysfunction [3, 4]. Moreover, methylmalonyl-CoA is an intermediate in the metabolism of propionic acid (PA), which is produced as a result of beta-oxidation of odd-chain fatty acids and branchedchain amino acids, metabolism of cholesterol sidechains, and fermentation by colonic microbiota $[5,6]$.

Thus, circulating concentrations of MMA depend to a certain extent on plasma vitamin B12 level and renal function but may also reflect endogenous production of PA. Importantly, PA has been found to be highly toxic [7]. Studies with PA in isolated hepatocytes suggest that toxicity of PA is due to accumulation of propionyl-CoA and methylmalonyl-CoA [7]. Intracellular accumulation of propionyl-CoA and methylmalonyl-CoA was found to be associated with impairment of several hepatic metabolic pathways, including gluconeogenesis, ureagenesis, pyruvate oxidation, and fatty acid oxidation [7].

In the present study, we first investigated to what extent MMA levels are explained by plasma vitamin B12 and eGFR. The variance in circulating MMA concentrations that remains unexplained by plasma vitamin B12 and eGFR may, at least in part, reflect PA production. We also investigated whether circulating MMA concentrations are associated with an increased risk for mortality and whether this association is independent of or interdependent with plasma vitamin B12 or eGFR.

\section{Methods}

\section{Study design and population}

The LifeLines Cohort Study is a large ongoing observational population-based cohort study that investigates health and health-related behaviors of more than 167,000 individuals. A detailed description of the Lifelines Cohort Study can be found elsewhere [8, 9]. Participants were recruited from the three Northern provinces of the Netherlands between 2006 and 2013. In short, the first group of participants was recruited via local general practices. Participants could indicate whether family members were interested as well. In addition, individuals who were interested in the study had the possibility to register via an online selfregistration. Individuals with insufficient knowledge of the Dutch language, with severe psychiatric or physical illness, and those with limited life expectancy $(<$ 5 years) were excluded from the study. Participants completed several questionnaires, including topics such as the occurrence of diseases, general health, medication use, diet, physical activity, and personality. Participants were invited to the Lifelines Research sites for a comprehensive health assessment and to allow storage of biological samples, including plasma, serum, and 24-h urine samples in the biobank underlying the LifeLines cohort. All participants provided written consent. The Lifelines Cohort Study was conducted according to the principles of the Declaration of Helsinki and approved by the Medical ethical committee of the University Medical Center Groningen, the Netherlands (METc approval number 2007/152).

The present study included individuals of the LifeLines-MINUTHE (MIcroNUTrients and Health disparities in Elderly) subcohort of the LifeLines Cohort Study. This subcohort consists of 1605 individuals aged between 60 and 75 years, with available plasma, serum, and 24-h urine samples from the biobank of the LifeLines cohort. The 1605 individuals comprised 400 men and 403 women with low socioeconomic status (SES) and 402 men and 400 women with high SES. Since education is more differentiating than income in the Dutch population, the classification of SES was based on educational status. Low SES was defined as never been to school or elementary school only, or completed lower vocational or secondary schooling; high SES was defined as completed higher vocational schooling or education. In the present study, we included 1533 individuals with available MMA, vitamin B12, and eGFR measurements.

\section{Data collection and measurements}

Data regarding demographics, education, smoking status, and general health were collected from self-administered questionnaires. Anthropometric measurements and blood pressure were measured by well-trained staff. BMI was calculated as weight $(\mathrm{kg})$ divided by height squared $\left(\mathrm{m}^{2}\right)$. Systolic and diastolic blood pressures were measured 10 times during a period of $10 \mathrm{~min}$ using an automated Dinamap Monitor (GE Healthcare, Freiburg, Germany). The average of the final three readings was used for each blood pressure parameter.

Blood samples were collected in a fasting state between 8.00 and 10.00 a.m. and subsequently transported to the Central Lifelines Laboratory in the University Medical Center Groningen. MMA was measured using LC-MS/MS. Vitamin B12 was measured using an electrochemiluminiscence immunoassay on a Roche Cobas chemistry analyzer (Roche, Mannheim, Germany). Serum creatinine (SCr) was measured via an enzymatic assay with colorimetric detection on a Roche Cobas 
chemistry analyzer (Roche, Mannheim, Germany). The creatinine-based CKD-EPI formula was used to obtain the estimated glomerular filtration rate (eGFR) [10]. Other laboratory measurements, including plasma total homocysteine, were assessed by commercially available assays on a Roche Cobas chemistry analyzer (Roche, Mannheim, Germany).

\section{Clinical endpoints}

In the present study, we investigated the association of MMA with all-cause mortality. Data on mortality were obtained from the municipal register.

\section{Statistical analyses}

Statistical analyses were performed using SPSS version 25 for Windows (IBM Corporation, Chicago, IL), STATA version 13.1 (StataCorp LP, TX, USA), and R version 3.5.2 (R Core Team (2017); R: A language and environment for statistical computing; R Foundation for Statistical Computing, Vienna, Austria; URL https://Rproject.org/). Results were expressed as mean \pm standard deviation (SD) or median (interquartile range) for normally and non-normally distributed data, respectively. Nominal data were presented as the total number of patients (percentage). A two-sided $P<0.05$ was considered to indicate statistical significance.

Baseline characteristics are presented for the total study population and for tertiles of baseline MMA concentrations. $P$ values for differences between tertiles were assessed using ANOVA for normally distributed data, Kruskal-Wallis test for skewed data, and the $\chi^{2}$ test for nominal data.

We used linear regression analyses to investigate the cross-sectional associations of MMA with vitamin B12, eGFR, and other parameters including SES. Logarithmic transformation of variables was used to fulfill criteria for linear regression analyses if necessary. First, univariable linear regression analyses were conducted. In addition, we tested for interactions between variables using multivariable linear regression analyses. Finally, multivariable linear regression models were developed using stepwise backward selection, without and with inclusion of the interaction term for vitamin B12 and eGFR (model 1 and model 2, resp.). Variable exclusion in the backward stepwise selection procedure was set at a $P$ value of 0.2 ; the $P$ value for subsequent variable inclusion was set to 0.05 . Results for variables with a $P$ value of $>0.2$ in univariable and multivariable linear regression analyses were not shown. $R^{2}$ and adjusted $R^{2}$ values were obtained to assess the proportion of variability in the data accounted for by single variables and the multivariable models. The $\mathrm{R}$ package plot3D was used to depict the cross-sectional interaction between vitamin B12 and eGFR with MMA levels.
We used Cox regression analyses to investigate the prospective association of MMA with all-cause mortality. We applied a $\log _{2}$ transformation of MMA values so the hazard ratios were expressed as an increase in risk per doubling of baseline MMA values. Cox regression analyses were also used to test for interaction between MMA and eGFR. Various Cox regression models were built to adjust for possible confounders. The first model depicts the interaction between $\log _{2}$ MMA and eGFR for the risk of mortality; model 2 was adjusted for age and sex; and model 3 was additionally adjusted for SES, smoking, alcohol intake, BMI, SBP, vitamin B12, and use of vitamin. In secondary analyses, we investigated whether prospective associations for MMA were paralleled by prospective associations for plasma total homocysteine. Model 1A and model 1B depict the interaction between $\log _{2}$ total plasma homocysteine and eGFR with respect to mortality and the interaction between $\log _{2}$ MMA and eGFR with respect to mortality; model 2A and model $2 \mathrm{~B}$ were fully adjusted for potential confounders, with additional adjustment for $\log _{2}$ MMA in case of the analysis for total plasma homocysteine (model 2A) and additional adjustment for $\log _{2}$ total plasma homocysteine in case of the analysis for MMA (model 2B); and model 3 was fully adjusted for potential confounders and included both the interaction between $\log _{2}$ total plasma homocysteine and eGFR with respect to mortality and the interaction between $\log _{2}$ MMA and eGFR with respect to mortality in one model. The assumption of proportional hazards was investigated by inspecting the Schoenfeld residuals. The $\mathrm{R}$ package plot3D was used to depict the interaction of MMA and eGFR in their association with mortality. As sensitivity analyses, we stratified Cox regression analyses for SES. In addition, we repeated Cox regression analyses after exclusion of subjects that used multivitamin or vitamin B supplements.

\section{Results}

\section{Baseline characteristics}

In this study, we included 1533 individuals. Baseline characteristics of the total study population and according to tertiles of MMA concentrations are presented in Table 1. At baseline, median MMA concentration was 170 (range 63-4638) nmol/L, median vitamin B12 concentration was 290 (range 64-1476) $\mathrm{pmol} / \mathrm{L}$ ), and median eGFR was 84 (range 19-109) $\mathrm{mL} / \mathrm{min} / 1.73 \mathrm{~m}^{2}$. A total of 104 individuals (7\%) had an MMA level $>340 \mathrm{nmol} / \mathrm{L}, 52$ individuals (3\%) had a vitamin B12 level $<145 \mathrm{pmol} / \mathrm{L}$, and 166 individuals (11\%) had a vitamin B12>450 pmol/L. We found that age, low education, concentrations of vitamin B12, concentrations of total homocysteine, eGFR, 
Table 1 Baseline characteristics of the study population $(n=1533)$

\begin{tabular}{|c|c|c|c|c|c|}
\hline & \multirow{2}{*}{$\begin{array}{l}\text { All subjects } \\
(n=1533)\end{array}$} & \multicolumn{3}{|l|}{ Tertiles of MMA } & \multirow[t]{2}{*}{$P$ value } \\
\hline & & Tertile $1(n=511)$ & Tertile $2(n=511)$ & Tertile $3(n=511)$ & \\
\hline MMA (nmol/L) & $170(138-216)$ & $\leq 148$ & $148-196$ & $\geq 196$ & - \\
\hline Vitamin B12 (pmol/L) & $290(224-362)$ & $332(264-416)$ & $295(231-356)$ & $240(190-309)$ & $<0.001$ \\
\hline eGFR (mL/min/1.73 m²) & $84(74-91)$ & $86(77-92)$ & $83(75-91)$ & 81 (69-90) & $<0.001$ \\
\hline \multicolumn{6}{|l|}{ Demographics } \\
\hline Male sex $(n, \%)$ & $769(50)$ & $254(50)$ & $252(50)$ & $263(51)$ & 0.8 \\
\hline Age (years) & $65(62-69)$ & $64(62-68)$ & $65(62-69)$ & $65(63-69)$ & $<0.001$ \\
\hline Education & & & & & $<0.001$ \\
\hline Low $(n, \%)$ & $761(50)$ & $228(45)$ & $242(47)$ & $291(57)$ & \\
\hline $\operatorname{High}(n, \%)$ & $772(50)$ & $283(55)$ & $269(53)$ & $220(43)$ & \\
\hline Smoking $(n, \%)$ & $183(12)$ & $55(11)$ & $62(12)$ & $66(13)$ & 0.6 \\
\hline Alcohol consumption & & & & & 0.9 \\
\hline Non-drinker (n, \%) & $242(16)$ & $79(15)$ & $84(16)$ & $79(15)$ & \\
\hline$\leq 1$ drink/day $(n, \%)$ & $552(36)$ & $185(36)$ & $190(37)$ & $177(35)$ & \\
\hline 1-2 drinks/day $(n, \%)$ & $309(20)$ & $114(22)$ & $101(20)$ & $94(18)$ & \\
\hline$>2$ drinks/day $(n, \%)$ & $174(11)$ & $63(12)$ & $61(12)$ & $50(10)$ & \\
\hline Diabetes ( $n, \%)$ & $156(10)$ & $58(11)$ & $42(8)$ & $56(11)$ & 0.2 \\
\hline History of CVD $(n, \%)$ & $186(12)$ & $58(11)$ & $65(13)$ & $63(12)$ & 0.8 \\
\hline \multicolumn{6}{|l|}{ Clinical measurements } \\
\hline BMI $\left(\mathrm{kg} / \mathrm{m}^{2}\right)$ & $26.4(24.1-29.4)$ & $26.1(24.1-29.1)$ & $26.5(24.0-29.3)$ & $26.3(24.1-29.8)$ & 0.5 \\
\hline Systolic blood pressure (mmHg) & $134 \pm 18$ & $134 \pm 17$ & $134 \pm 17$ & $135 \pm 18$ & 0.6 \\
\hline Diastolic blood pressure (mmHg) & $75 \pm 9$ & $75 \pm 9$ & $75 \pm 9$ & $76 \pm 10$ & 0.2 \\
\hline \multicolumn{6}{|l|}{ Laboratory parameters } \\
\hline $\mathrm{Hb}(\mathrm{mmol} / \mathrm{L})$ & $8.8 \pm 0.7$ & $8.8 \pm 0.7$ & $8.8 \pm 0.7$ & $8.8 \pm 0.7$ & 0.6 \\
\hline $\mathrm{MCV}(\mathrm{fL})$ & $91 \pm 4$ & $91 \pm 4$ & $91 \pm 4$ & $91 \pm 4$ & 0.7 \\
\hline Total cholesterol-HDL ratio & $3.5(2.9-4.3)$ & $3.5(2.9-4.4)$ & $3.5(3.0-4.3)$ & $3.5(2.9-4.4)$ & 0.9 \\
\hline Triglycerides (mmol/L) & $1.1(0.8-1.5)$ & $1.1(0.8-1.5)$ & $1.1(0.8-1.5)$ & $1.1(0.8-1.5)$ & 0.4 \\
\hline Serum creatinine $(\mu \mathrm{mol} / \mathrm{L})$ & $75(66-85)$ & $73(64-84)$ & $75(66-84)$ & $78(67-88)$ & $<0.001$ \\
\hline Total homocysteine $(\mu \mathrm{mol} / \mathrm{L})$ & $13(11-15)$ & $12(11-14)$ & $13(11-15)$ & $14(12-17)$ & $<0.001$ \\
\hline \multicolumn{6}{|l|}{ Medication } \\
\hline Vitamin supplements $(n, \%)$ & $173(11)$ & $71(14)$ & $58(11)$ & $44(9)$ & 0.03 \\
\hline
\end{tabular}

$B M I$ body mass index, eGFR estimated glomerular filtration rate, $H b$ hemoglobin, $H D L$ high-density lipoprotein, $M M A$ methylmalonic acid

and use of vitamin supplements were significantly different across tertiles of MMA (Table 1).

\section{Determinants of MMA}

Results of univariable and multivariable linear regression analyses are depicted in Table 2 . We found a significant association of $\log _{2}$ vitamin B12, $\log _{2}$ eGFR, age, education, and use of vitamin supplements with $\log _{2}$ MMA in univariable linear regression analyses. $R^{2}$ values were highest for $\log _{2}$ vitamin B12 (0.180) and $\log _{2}$ eGFR (0.036). Furthermore, we found a significant interaction between $\log _{2}$ vitamin $\mathrm{B} 12$ and $\log _{2}$ eGFR in multivariable linear regression analyses $\left(P_{\text {interaction }}=0.02\right)$. The $R^{2}$ values for multivariable linear regression models 1 and 2 were 0.22 (Table 2). The cross-sectional association between vitamin B12, eGFR, and MMA is depicted in Fig. 1. As shown in Fig. 1, MMA concentrations are highest among subjects with the lowest vitamin B12 concentrations and lowest eGFR. These results did not materially change after the exclusion of subjects that used a multivitamin or vitamin B containing supplement (Additional files 1 and 2).

\section{MMA, eGFR, and mortality}

After a total follow-up time of 8.5 years (median 5.3 [4.3-6.5] years), 72 subjects had died. In univariable Cox regression analyses, we found a significant association of $\log _{2}$ MMA with mortality (HR 1.67 (95\% CI 1.25-2.22), 
Table 2 Univariable and multivariable linear regression analyses for $\log _{2}$ MMA

\begin{tabular}{|c|c|c|c|c|c|c|}
\hline & \multicolumn{2}{|c|}{ Univariable } & \multicolumn{2}{|c|}{$\begin{array}{l}\text { Multivariable } \\
\text { Model } 1\end{array}$} & \multicolumn{2}{|c|}{$\begin{array}{l}\text { Multivariable } \\
\text { Model } 2\end{array}$} \\
\hline & Std $b$ & $P$ value & Std $b$ & $P$ value & Std $b$ & $P$ value \\
\hline $\log _{2}$ vitamin B12 (pmol/L) & -0.424 & $<0.001$ & -0.434 & $<0.001$ & 1.131 & 0.08 \\
\hline $\log _{2}$ eGFR (mL/min/1.73 m²) & -0.189 & $<0.001$ & -0.162 & $<0.001$ & 0.689 & 0.048 \\
\hline $\log _{2}$ vitamin $B 12 \times \log _{2} e G F R$ & - & - & - & - & -1.796 & 0.01 \\
\hline \multicolumn{7}{|l|}{ Demographics } \\
\hline Male sex & 0.002 & 0.9 & -0.065 & 0.005 & -0.053 & 0.02 \\
\hline Age (years) & 0.093 & $<0.001$ & 0.050 & 0.04 & 0.034 & 0.2 \\
\hline High education & -0.099 & $<0.001$ & - & - & -0.030 & 0.2 \\
\hline \multicolumn{7}{|l|}{ Medication } \\
\hline Use of vitamin supplements & -0.069 & 0.007 & - & - & - & - \\
\hline
\end{tabular}

Model 1: $R^{2}=0.22 ;$ adjusted $R^{2}=0.22$

Model 2: $R^{2}=0.22$; adjusted $R^{2}=0.21$

eGFR estimated glomerular filtration rate, MMA methylmalonic acid

$P<0.001)$ and eGFR (per $10 \mathrm{~mL} / \mathrm{min} / 1.73 \mathrm{~m}^{2}$ ) with mortality (0.73 (0.62-0.85), $P<0.001)$, while $\log _{2}$ vitamin B12 was not significantly associated with mortality (0.74 (0.49-1.11), $P=0.1)$. Moreover, we found a significant interaction between MMA and eGFR with respect to mortality, which remained significant independent of adjustment for potential confounders (Table 3). The interaction between MMA and eGFR with respect to mortality is depicted in Fig. 2. Figure 2 shows that higher MMA values as well as lower eGFR values are associated with an increased risk for all-cause mortality and that this mortality risk strongly increases when MMA levels increase and eGFR decreases.

In secondary analyses, we investigated whether these findings for MMA might be paralleled by similar associations for plasma total homocysteine. In these analyses, we found a similar interaction for $\log _{2}$ total plasma homocysteine and eGFR with respect to mortality as for $\log _{2}$ MMA and eGFR with respect to mortality, albeit somewhat weaker for the former interaction compared to the latter

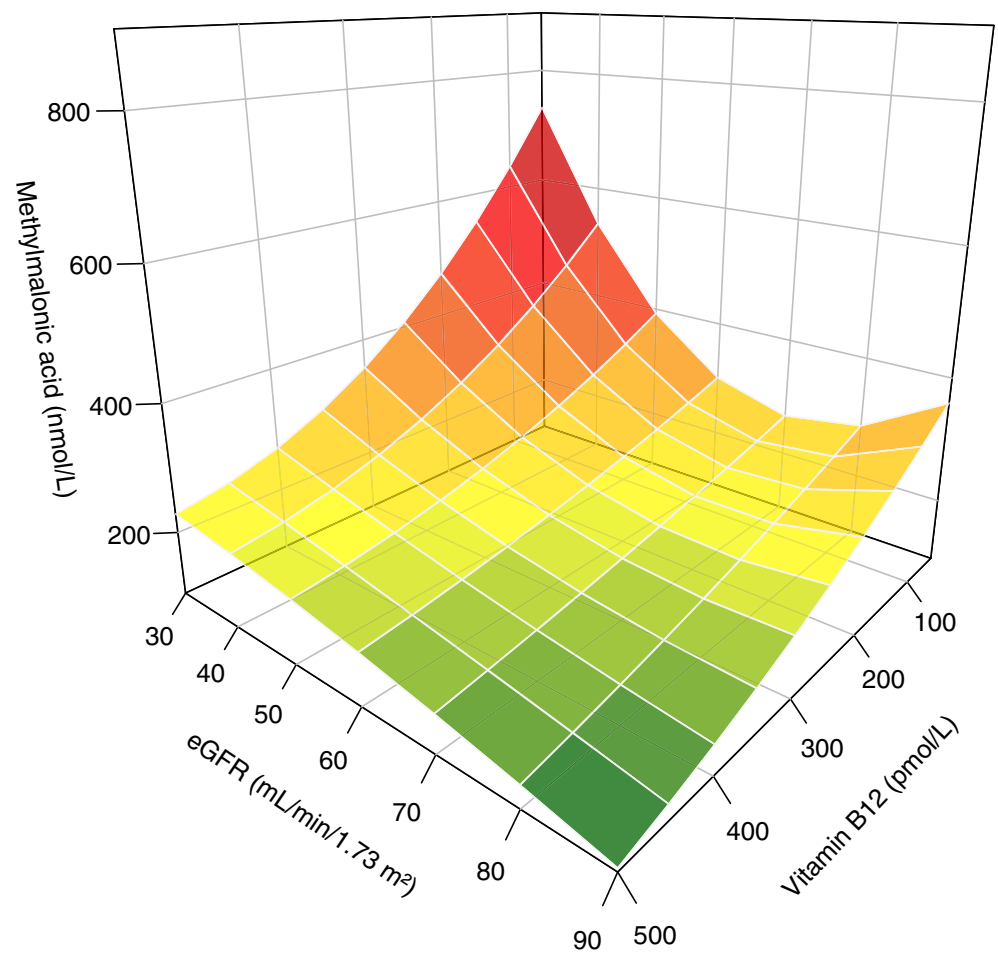

Fig. $13 \mathrm{D}$ plot depicting the unadjusted cross-sectional association between methylmalonic acid, vitamin B12, and eGFR 
Table 3 Prospective associations of $\log _{2}$ MMA, eGFR, and their interaction term, with all-cause mortality

\begin{tabular}{|c|c|c|c|c|c|c|}
\hline & \multicolumn{2}{|l|}{ Model 1} & \multicolumn{2}{|l|}{ Model 2} & \multicolumn{2}{|l|}{ Model 3} \\
\hline & HR $(95 \% \mathrm{Cl})$ & $\overline{P \text { value }}$ & HR (95\% Cl) & $\overline{P \text { value }}$ & HR $(95 \% \mathrm{Cl})$ & $P$ value \\
\hline $\log _{2}$ MMA (nmol/L) & $13.33(3.87-45.91)$ & $<0.001$ & $15.24(4.47-52.03)$ & $<0.001$ & $11.48(3.32-39.64)$ & $<0.001$ \\
\hline eGFR $\left(10 \mathrm{~mL} / \mathrm{min} / \mathrm{m}^{2}\right)$ & $7.36(1.98-27.41)$ & 0.003 & $9.79(2.61-36.70)$ & 0.001 & $7.57(1.98-28.94)$ & 0.003 \\
\hline $\log _{2} M M A \times e G F R$ & $0.76(0.65-0.89)$ & 0.001 & $0.74(0.64-0.87)$ & $<0.001$ & $0.77(0.65-0.90)$ & 0.001 \\
\hline
\end{tabular}

Model 1: $\log _{2}$ MMA, eGFR, $\log _{2}$ MMA $\times$ eGFR

Model 2: adjusted for age and sex

Model 3: as model 2 + SES, smoking, alcohol intake, BMI, SBP, vitamin B12, and use of vitamin supplements

$N_{\text {events }} / n_{\text {total }}=72 / 1533$

$B M I$ body mass index, eGFR estimated glomerular filtration rate, SBP systolic blood pressure, SES socioeconomic status

interaction (Additional file 3, model 1A and model 1B, resp.). In models in which the interactions were adjusted for potential confounders, we applied additional adjustment for $\log _{2}$ MMA in case of the analysis for total plasma homocysteine (Additional file 3, model 2A), and we applied additional adjustment for $\log _{2}$ total plasma homocysteine in case of the analysis for MMA (Additional file 3 , model $2 \mathrm{~B}$ ). In these analyses, the results were also consistent with a parallel interaction for both parameters, albeit again somewhat weaker for the interaction between plasma total homocysteine and eGFR than for the interaction between MMA and eGFR. In further analyses, in which we included both interactions in one fully adjusted model, the interaction between $\log _{2}$ plasma total homocysteine and eGFR lost significance $\left(0.98\right.$ (0.77-1.26), $P_{\text {in- }}$ teraction $=0.9)$, while the interaction between $\log _{2}$ MMA and eGFR remained $\left(0.79(0.65-0.97), P_{\text {interaction }}=0.03\right)$ (Additional file 3, model 3).

As sensitivity analyses, we stratified Cox regression analyses for SES. The results of these sensitivity analyses were not materially different from the Cox regression analyses in which we adjusted for SES (Additional file 4). Furthermore, analyses were repeated after exclusion of subjects that used multivitamin or vitamin B supplements at baseline. The results did not materially change after exclusion of subjects that used multivitamin or vitamin B supplements at baseline (Additional files 5 and 6).

\section{Discussion}

In this study, we found that vitamin B12 and eGFR were significantly associated with MMA levels. However, only $22 \%$ of the variation in MMA values was explained by

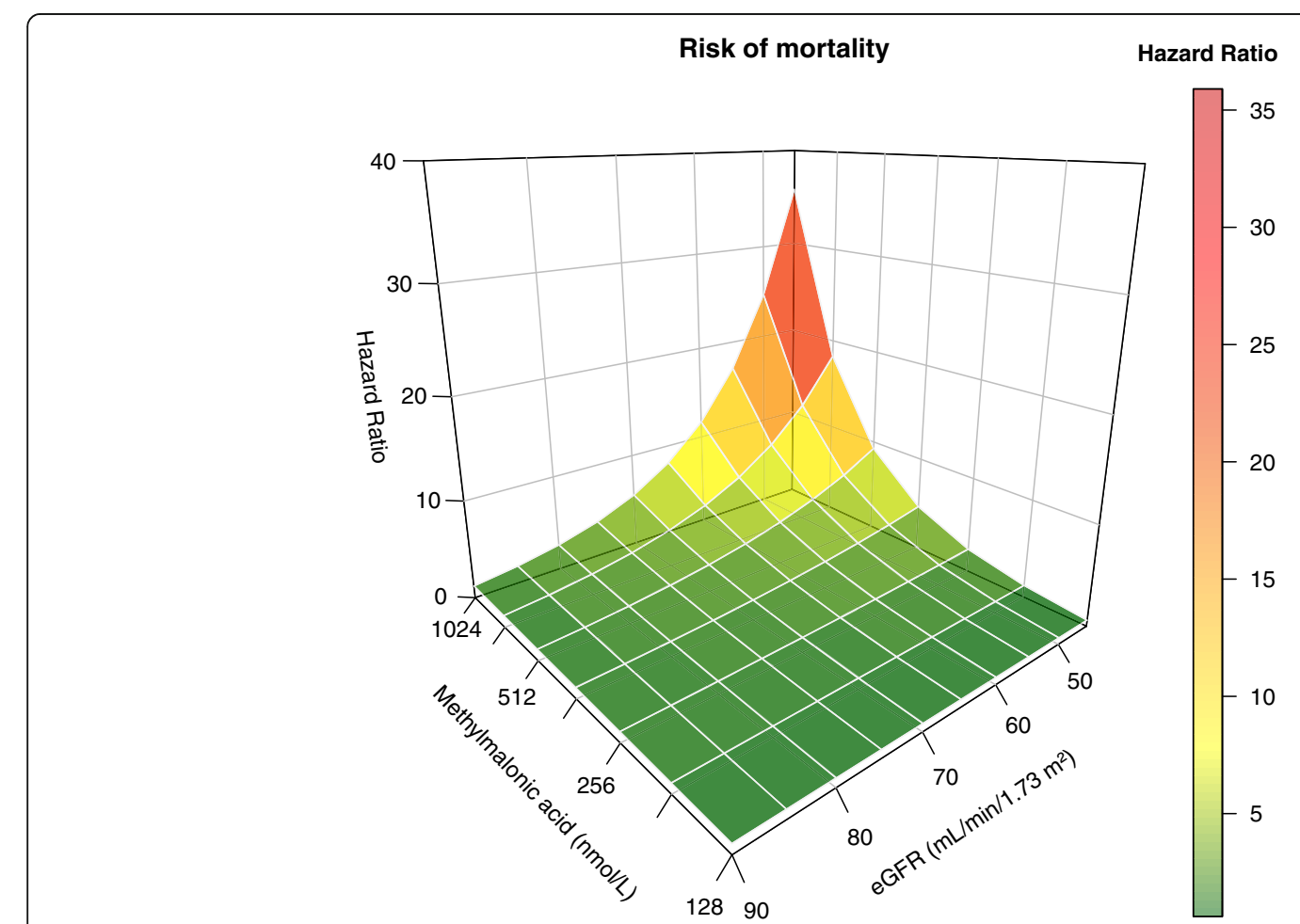

Fig. 2 3D plot depicting the unadjusted interaction between methylmalonic acid and eGFR with all-cause mortality 
vitamin B12, eGFR, age, and sex, indicating that a large part of variation in MMA levels was attributable to factors other than vitamin B12 and eGFR. Furthermore, to the best of our knowledge, we are the first to demonstrate that elevated MMA levels are associated with an increased risk for mortality. We found a significant interaction between MMA and eGFR in relation to mortality, indicating that this association was more pronounced in individuals with impaired renal function. These findings are of particular interest since MMA is a potentially modifiable marker, not only through pharmacological intervention, but also through dietary interventions, and could therefore be an important tool for guiding the improvement of health and longevity in the general population.

In this study, we investigated the cross-sectional association of vitamin B12 and eGFR with MMA. In line with previous studies [2-4], we found that MMA levels are associated with vitamin B12 and eGFR and that MMA levels are highest in subjects with a combination of low vitamin B12 levels and impaired renal function. However, we found that only $22 \%$ of variation in circulating MMA concentration was explained by vitamin B12, eGFR, age, sex, and SES. This finding is in line with a previous study from Vogiatzoglou et al. that demonstrated that $16 \%$ of variation in MMA was explained by vitamin B12, plasma creatinine, and sex [2]. Furthermore, Vogiatzoglou et al. could also not identify factors other than age, plasma creatinine, vitamin B12, and sex that substantially influenced plasma MMA [2]. The addition of anthropometric measures, lifestyle, and dietary factors (i.e., dietary intake of food items such as meat and fish) did not substantially add to the explained variation of MMA $\left(R^{2}=0.167\right)[2]$.

As stated previously, MMA is an intermediate in the metabolism of PA $[5,6]$. The main source of PA is catabolism of the amino acids valine, isoleucine, methionine, and threonine, and other pathways including catabolism of odd-chain fatty acids and cholesterol sidechains [11]. Furthermore, PA is also produced by anaerobic fermentation of carbohydrates and other compounds within the gut, from which it is absorbed into the portal circulation [11, 12]. Although there are wide individual variations in PA production from different sources, stable isotope studies in patients with propionic and methylmalonic acidemia indicate that approximately $50 \%$ of PA production is derived from amino acid catabolism, 25\% from anaerobic fermentation in the gut, and $25 \%$ from catabolism of odd-chain fatty acids [11, 13, 14]. Thus, variation in plasma MMA concentrations in the absence of vitamin B12 deficiency or renal dysfunction may also result from increased production of PA and its metabolites or decreased renal clearance thereof.
Importantly, to the best of our knowledge, this study is the first to demonstrate that elevated MMA levels are associated with an increased risk for all-cause mortality. Moreover, we found that this association was more pronounced in individuals with impaired renal function. Since the present study is an observational, and not a mechanistic, study, we can only speculate on possible underlying pathophysiological mechanisms for this association. First, elevated MMA may be a marker of abnormal gut microbiota, in particular high PA bacteria. It is known that chronic kidney disease (CKD) itself, together with CKD-related changes in diet and medication, can induce changes in both the composition and metabolic activity of gut microbiota [15]. These CKD-related changes in microbiota may also affect metabolic and cardiovascular health by secretion of metabolites that favor insulin resistance, obesity, endothelial dysfunction, and cardiovascular aging [15], which may explain the increased mortality risk in individuals with impaired renal function. In addition, since a large part of PA production is derived from catabolism of amino acids and odd-chain fatty acids $[11,13,14]$, catabolism related to chronic diseases may also be a possible underlying mechanism [16]. It is known that catabolism is related with an increased morbidity and mortality risk in CKD $[17,18]$, which also might be a possible explanation for the strongly increased mortality risk in individuals with impaired renal function. In secondary analyses, we found that the interaction between MMA and eGFR with respect to mortality was paralleled by a similar, albeit less strong, interaction between plasma total homocysteine and eGFR with respect to mortality, which is suggestive of parallelism in mechanisms underlying prospective associations of MMA and plasma total homocysteine with respect to mortality.

Some limitations of the present study need to be addressed. First, given the unavailability of PA measurements, we were not able to investigate the association between MMA and PA levels. Additional studies are needed to investigate the association between MMA and PA and other potential factors influencing MMA levels. Second, given the observational nature of this study, it is impossible to draw a definite conclusion about the causality of the association of MMA with mortality. In addition, given the observational nature, we can only speculate on potential pathophysiological pathways. Further studies are needed to investigate whether the association between MMA and mortality is causal and to provide more insight in the underlying pathophysiological pathways, especially in subjects with chronic kidney disease. Third, we had no data available on the cause of death. It has been suggested that the mechanistic association between vitamin B12 and MMA can be disrupted in patients with advanced stages of malignant 
disease [19, 20]. Although we excluded subjects with known malignant disease at baseline, residual confounding by previously unrecognized malignant disease contributing to premature death during follow-up remains a possibility. A major strength of this study is that it is the first to investigate the prospective association of MMA levels with mortality. Additional strengths are the prospective study design, the large sample size, availability of MMA measurements, and follow-up data on mortality.

\section{Conclusions}

In conclusion, we found that vitamin B12 and eGFR were significantly associated with MMA levels, but only explained a small part (i.e., 22\%) of the variation in MMA values. In addition, we found that higher MMA levels are independently associated with an increased risk for mortality in a general population cohort. Moreover, we found a significant interaction between MMA, eGFR, and mortality, indicating that this association was more pronounced in individuals with impaired renal function.

\section{Supplementary Information}

Supplementary information accompanies this paper at https://doi.org/10. 1186/s12916-020-01853-x.

Additional file 1 Univariable and multivariable linear regression analyses for $\log _{2}$ MMA after exclusion of individuals that used multivitamin or vitamin B supplements $(n=1360)$.

Additional file 2. 3D plot depicting the unadjusted cross-sectional association between methylmalonic acid, vitamin B12 and eGFR after exclusion of individuals that used multivitamin or vitamin B supplements.

Additional file 3. Parallel comparison of prospective analyses of associations of $\log _{2}$ plasma total homocysteine, $\log _{2}$ MMA and their interactions with eGFR with respect to all-cause mortality.

Additional file 4. Prospective associations of $\log _{2} M M A$, eGFR and of interaction of $\log _{2}$ MMA with eGFR, respectively, with all-cause mortality stratified for SES ( $n_{\text {events }} / n_{\text {total }}=72 / 1533$ ).

Additional file 5. Interaction of $\log _{2}$ MMA with eGFR and all-cause mortality after exclusion of individuals that used multivitamin or vitamin $B$ supplements ( $n_{\text {events }} / n_{\text {total }}=68 / 1360$ ).

Additional file 6. 3D plot depicting the unadjusted interaction between methylmalonic acid and eGFR with all-cause mortality after exclusion of individuals that used multivitamin or vitamin B supplements.

\section{Acknowledgements}

Not applicable.

\section{Authors' contributions}

SB designed the research. IM and SB acquired the data. IR performed the statistical analysis and drafted the manuscript. IM, DG, and SB contributed to the statistical analysis and interpretation of the data. AP, ME, JK, MB, GN, FM, $\mathrm{IK}, \mathrm{RH}$, and SB provided critical review, advice, and consultation throughout. All authors read and approved the final manuscript.

\section{Funding}

This research was funded by Top Institute Food and Nutrition (grant $\mathrm{CH}-003$ ). The funders had no role in study design, data collection and analysis, decision to publish, or preparation of the manuscript.

\section{Availability of data and materials}

All data generated or analyzed during this study are included in this published article [and its supplementary information files].

\section{Ethics approval and consent to participate}

All participants provided written consent. The Lifelines Cohort Study was conducted according to the principles of the Declaration of Helsinki and approved by the Medical ethical committee of the University Medical Center Groningen, the Netherlands (METc approval number 2007/152).

\section{Consent for publication}

Not applicable.

\section{Competing interests}

The authors declare that they have no competing interests.

\section{Author details}

${ }^{1}$ Department of Laboratory Medicine, University of Groningen, University Medical Center Groningen, P.O. Box 30.001, 9700 RB Groningen, The Netherlands. ${ }^{2}$ Department of Internal Medicine, Division of Nephrology, University of Groningen, University Medical Center Groningen, Groningen, The Netherlands. ${ }^{3}$ DSM Nutritional Products, Kaiseraugst, Switzerland.

Received: 26 July 2020 Accepted: 11 November 2020

Published online: 10 December 2020

\section{References}

1. Klee GG. Cobalamin and folate evaluation: measurement of methylmalonic acid and homocysteine vs vitamin B (12) and folate. Clin Chem. 2000;46(8 Pt 2):1277-83

2. Vogiatzoglou A, Oulhaj A, Smith AD, Nurk E, Drevon CA, Ueland PM, Vollset SE, Tell GS, Refsum H. Determinants of plasma methylmalonic acid in a large population: implications for assessment of vitamin B12 status. Clin Chem. 2009:55(12):2198-206

3. Herrmann W, Schorr H, Geisel J, Riegel W. Homocysteine, cystathionine, methylmalonic acid and B-vitamins in patients with renal disease. Clin Chem Lab Med. 2001;39(8):739-46.

4. van Loon SL, Wilbik AM, Kaymak U, van den Heuvel ER, Scharnhorst V, Boer AK. Improved testing for vitamin B12 deficiency: correcting MMA for eGFR reduces the number of patients classified as vitamin B12 deficient. Ann Clin Biochem. 2018:55(6):685-92.

5. Al-Lahham SH, Peppelenbosch MP, Roelofsen H, Vonk RJ, Venema K. Biological effects of propionic acid in humans; metabolism, potential applications and underlying mechanisms. Biochim Biophys Acta. 2010; 1801(11):1175-83

6. Hosseini E, Grootaert C, Verstraete W, Van de Wiele T. Propionate as a health-promoting microbial metabolite in the human gut. Nutr Rev. 2011; 69(5):245-58.

7. Krahenbuhl S, Brass EP. Inhibition of hepatic propionyl-CoA synthetase activity by organic acids. Reversal of propionate inhibition of pyruvate metabolism. Biochem Pharmacol. 1991;41(6-7):1015-23.

8. Klijs B, Scholtens S, Mandemakers JJ, Snieder H, Stolk RP, Smidt N Representativeness of the LifeLines Cohort Study. PLoS One. 2015;10(9): e0137203.

9. Scholtens S, Smidt N, Swertz MA, Bakker SJ, Dotinga A, Vonk JM, van Dijk F, van Zon SK, Wijmenga C, Wolffenbuttel BH, Stolk RP. Cohort profile: LifeLines, a three-generation cohort study and biobank. Int J Epidemiol. 2015:44(4):1172-80.

10. Levey AS, Stevens LA, Schmid CH, Zhang YL, Castro AF,3rd, Feldman HI, Kusek JW, Eggers P, Van Lente F, Greene T, Coresh J, CKD-EPI (Chronic Kidney Disease Epidemiology Collaboration): a new equation to estimate glomerular filtration rate. Ann Intern Med 2009, 150(9):604-612.

11. Leonard JV. Stable isotope studies in propionic and methylmalonic acidaemia. Eur J Pediatr. 1997;156(Suppl 1):S67-9.

12. Louis P, Flint HJ. Formation of propionate and butyrate by the human colonic microbiota. Environ Microbiol. 2017;19(1):29-41.

13. Sba Ã ${ }^{-}$D, Narcy C, Thompson GN, Mariotti A, Poggi F, Saudubray JM, Bresson JL: Contribution of odd-chain fatty acid oxidation to propionate production in disorders of propionate metabolism. Am J Clin Nutr 1994, 59(6):1332-1337. 
14. Thompson GN, Walter JH, Bresson JL, Ford GC, Lyonnet SL, Chalmers RA, Saudubray JM, Leonard JV, Halliday D. Sources of propionate in inborn errors of propionate metabolism. Metabolism. 1990;39(11):1133-7.

15. Meijers B, Evenepoel $\mathrm{P}$, Anders $\mathrm{HJ}$. Intestinal microbiome and fitness in kidney disease. Nat Rev Nephrol. 2019;15(9):531-45.

16. Nie C, He T, Zhang W, Zhang G, Ma X: Branched chain amino acids: beyond nutrition metabolism. Int J Mol Sci 2018, 19(4):https://doi.org/10.3390/ ijms19040954.

17. Gracia-Iguacel C, Gonzalez-Parra E, Perez-Gomez MV, Mahillo I, Egido J, Ortiz A, Carrero JJ. Prevalence of protein-energy wasting syndrome and its association with mortality in haemodialysis patients in a Centre in Spain Nefrologia. 2013;33(4):495-505.

18. Wang XH, Mitch WE. Mechanisms of muscle wasting in chronic kidney disease. Nat Rev Nephrol. 2014;10(9):504-16.

19. Vashi P, Edwin P, Popiel B, Lammersfeld C, Gupta D. Methylmalonic acid and homocysteine as indicators of vitamin B-12 deficiency in cancer. PLoS One. 2016;11(1):e0147843.

20. Solomon LR. Functional vitamin B12 deficiency in advanced malignancy implications for the management of neuropathy and neuropathic pain. Support Care Cancer. 2016;24(8):3489-94.

\section{Publisher's Note}

Springer Nature remains neutral with regard to jurisdictional claims in published maps and institutional affiliations.

Ready to submit your research? Choose BMC and benefit from:

- fast, convenient online submission

- thorough peer review by experienced researchers in your field

- rapid publication on acceptance

- support for research data, including large and complex data types

- gold Open Access which fosters wider collaboration and increased citations

- maximum visibility for your research: over $100 \mathrm{M}$ website views per year

At BMC, research is always in progress.

Learn more biomedcentral.com/submissions 